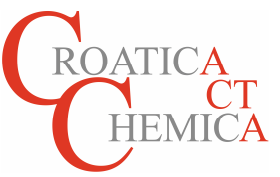

\title{
Comparison Between Two Eccentricity-based Topological Indices of Graphs
}

\author{
Kexiang Xu, * Xia Li,
}

\author{
College of Science, Nanjing University of Aeronautics \& Astronautics, Nanjing, Jiangsu 210016, PR China \\ * Corresponding author's e-mail address: kexxu1221@126.com \\ \# Corresponding author's e-mail address: nhlxylixia@163.com
}

RECEIVED: October 20, 2016 * REVISED: February 16, 2017 * ACCEPTED: February 17, 2017

THIS PAPER IS DEDICATED TO PROFESSOR NENAD TRINAJSTIĆ ON THE OCCASION OF HIS 80 ${ }^{\text {TH }}$ BIRTHDAY

\begin{abstract}
For a connected graph $G$, the eccentric connectivity index (ECl) and the first Zagreb eccentricity index of $G$ are defined as $\xi^{c}(G)=\sum_{v_{i \in V(G)}} \operatorname{deg}_{G}\left(v_{i}\right) \varepsilon_{G}\left(v_{i}\right)$ and $E_{1}(G)=\sum_{v_{i} \in V(G)} \varepsilon_{G}\left(v_{i}\right)^{2}$, respectively, where $\operatorname{deg}_{G}\left(v_{i}\right)$ is the degree of $v_{i}$ in $G$ and $\varepsilon_{G}\left(v_{i}\right)$ denotes the eccentricity of vertex $v_{i}$ in $G$. In this paper we compare the eccentric connectivity index and the first Zagreb eccentricity index of graphs. It is proved that $E_{1}(T)>\xi^{c}(T)$ for any tree $T$. This improves a result by Das ${ }^{[25]}$ for the chemical trees. Moreover, we also show that there are infinite number of chemical graphs $G$ with $E_{1}(G)>\xi^{c}(G)$. We also present an example in which infinite graphs $G$ are constructed with $E_{1}(G)=\xi^{c}(G)$ and give some results on the graphs $G$ with $E_{1}(G)<\xi^{c}(G)$. Finally, an effective construction is proposed for generating infinite graphs with each comparative inequality possibility between these two topological indices.
\end{abstract}

Keywords: Graph, First Zagreb eccentricity index, Eccentric connectivity index.

\section{INTRODUCTION}

W E only consider finite, undirected and simple graphs throughout this paper. Let $G$ be a graph with vertex set $V(G)=\left\{v_{1}, v_{2}, \ldots \ldots, v_{n}\right\}$ and edge set $E(G)$. The degree of $v_{i} \in V(G)$, denoted by $\operatorname{deg}_{G}\left(v_{i}\right)$, is the number of vertices in $G$ adjacent to $v$. For any two vertices $v_{i}, v_{j}$ in a graph $G$, the distance between them, denoted by $d_{G}\left(v_{i}, v_{j}\right)$, is the length of a shortest path connecting them in $G$. Other undefined notations and terminology on the graph theory can be found in. ${ }^{[1]}$

For any vertex of graph $G$, the eccentricity $\varepsilon_{G}\left(v_{i}\right)$ is the maximum distance from $v_{i}$ to other vertices of $G$, i.e., $\varepsilon_{G}\left(v_{i}\right)=\max d_{G}\left(v_{i}, v_{j}\right)$. If $\varepsilon_{G}\left(v_{i}\right)=d_{G}\left(v_{i}, v_{j}\right)$, then $v_{j}$ is an eccentric vertex of vertex $v_{i}$. For any graph $G$, we denote by $\bar{G}$ the complement of $G$. As usual, let $S_{n}, P_{n}, C_{n}, K_{n}$ be the star graph, path graph, cycle graph and complete graph, respectively, on $n$ vertices. We denote by $K_{n_{1}, n_{2}}$ the complete bipartite graph with bipartition of sizes $n_{1}$ and $n_{2}$. The Cartesian product $G \square H$ of graphs $G$ and $H$ is the graph with $V(G \square H)=V(G) \times V(H)$ and $(g, h)$ is adjacent to $\left(g^{\prime}, h^{\prime}\right)$ if and only if $g g^{\prime} \in E(G)$ and $h=h^{\prime}$, or $g=g^{\prime}$ and $h h^{\prime} \in E(H)$. If $G=H$, then $G \square H$ is denoted by $G^{(2)}$ for short. Moreover, $G^{(k)}$ can be similarly defined.

A graphical invariant is a number related to a graph which is a structural invariant, in other words, it is a fixed number under graph automorphisms. In chemical graph theory, these invariants are also known as the topological indices. Two of the oldest graph invariants are the wellknown Zagreb indices first introduced in Ref. [2] where Gutman and Trinajstić examined the dependence of total $\pi$-electron energy on molecular structure and elaborated in Ref. [3] For a (molecular) graph $G$, the first Zagreb index $M_{1}(G)$ and the second Zagreb index $M_{2}(G)$ are, respectively, defined as follows:

$$
\begin{aligned}
& M_{1}=M_{1}(G)=\sum_{v_{i} \in V(G)} \operatorname{deg}_{G}\left(v_{i}\right)^{2}, \\
& M_{2}=M_{2}(G)=\sum_{v_{i} v_{j} \in E(G)} \operatorname{deg}_{G}\left(v_{i}\right) \operatorname{deg}_{G}\left(v_{j}\right) .
\end{aligned}
$$

These two classical topological indices reflect the extent of branching of the molecular carbon-atom skeleton. ${ }^{[4]}$ The main properties of $M_{1}$ and $M_{2}$ were summarized in Refs. $[5,6]$. Other recent results on Zagreb indices can be found in Ref. [7] and the references cited therein. 
In analogy with the first and second Zagreb indices of graphs, some variants of them are invented, such as multiplicative Zagreb indices, ${ }^{[8,9]}$ multiplicative sum Zagreb index, ${ }^{[10,11]}$ Zagreb coindex ${ }^{[12,13]}$ and so on. In particular, Vukičević and Graovac ${ }^{[14]}$ defined the first and second Zagreb eccentricity indices as follows:

$$
E_{1}(G)=\sum_{v_{i} \in V(G)} \varepsilon_{G}\left(v_{i}\right)^{2}, E_{2}(G)=\sum_{v_{i} v_{j} \in E(G)} \varepsilon_{G}\left(v_{i}\right) \varepsilon_{G}\left(v_{j}\right) .
$$

Some mathematical properties of $E_{1}$ and $E_{2}$ can be found in Refs. [15,16].

In 1997, Sharma, Goswami and Madan ${ }^{[17]}$ introduced a distance-based molecular structure descriptor, which is named as "eccentric connectivity index" and defined as

$$
\xi^{c}(G)=\sum_{v_{i} \in V(G)} \operatorname{deg}_{G}\left(v_{i}\right) \varepsilon_{G}\left(v_{i}\right)
$$

The eccentric connectivity index (ECI) has been employed successfully for the development of numerous mathematical models for the prediction of biological activities of diverse nature. ${ }^{[18-20]} \mathrm{The} \mathrm{ECl}$ also can be written as follows:

$$
\xi^{c}(G)=\sum_{v_{i} v_{j} \in E(G)}\left(\varepsilon\left(v_{i}\right)+\varepsilon\left(v_{j}\right)\right)
$$

Some properties of ECl have been reported in Refs. [21-23].

A tree with maximum degree at most 4 is called chemical tree, which provides the graph representation of alkanes. ${ }^{[24]}$ In particular, a graph with maximum degree at most 4 is called chemical graph. Denote by $\mathcal{T}_{n}(d)$ the set of trees of order $n$ and with diameter $d$.

From definition, we have $E_{1}\left(S_{n}\right)>\xi^{c}\left(S_{n}\right)$ and $E_{1}\left(K_{n}\right)<\xi^{c}\left(K_{n}\right)$ for $n \geq 3$. Therefore these two topological indices $E_{1}$ and $\xi^{c}$ are incomparable. In Ref. [25], Das proved that $E_{1}(T)>\xi^{c}(T)$ for any chemical tree $T$. The paper is organized as follows. In Section 2, we show that $E_{1}(T)>\xi^{c}(T)$ for any tree $T$. And we present that there are infinite number of chemical graphs $G$ with $E_{1}(G)>\xi^{c}(G)$. In Section 3, we give an example in which infinite number of graphs $G$ are constructed with $E_{1}(G)=\xi^{c}(G)$. Also several sufficient conditions are proved for graphs $G$ with $E_{1}(G)<\xi^{c}(G)$. In Section 4, an effective construction is presented for generating infinite graphs with each comparison possibility between these two topological indices.

\section{THE GRAPHS WITH $E_{1}(G)>\xi^{c}(G)$}

In this section we characterize some graphs $G$ with $E_{1}(G)>\xi^{c}(G)$. Clearly, $E_{1}\left(P_{2}\right)=\xi^{c}\left(P_{2}\right)$. Then we deal with the case when $T$ is a tree of order $n \geq 3$. Next we will prove that there are infinite number of chemical graphs $G$ with $E_{1}(G)>\xi^{c}(G)$.
Before presenting the main results, we need to introduce some notations. A caterpillar, ${ }^{[26]}$ denoted by $P_{k+1}^{n}\left(a_{2}, a_{3}, \ldots, a_{k}\right)$ with $\sum_{i=2}^{k} a_{i}=n-k-1$, is a tree of order $n$ with diameter $k$ obtained from a path $P_{k+1}=v_{1} v_{2} \cdots v_{k+1}$ by attaching $a_{i} \geq 0$ pendant vertices to the vertex $v_{i}$ for $i=2,3, \ldots, k$. If $k$ is even, then $P_{k+1}$ has a unique central vertex $v_{k+1}$. Otherwise, $P_{k+1}$ has two adjacent central vertices $v_{k-1}^{\frac{k}{2}+1}$ and $v_{k+1}$. If $k=3$ in $P_{k+1}^{n}\left(a_{2}, a_{3}, \ldots, a_{k}\right)$ with $a_{2}=p \overline{21} \geq 0, \overline{a_{k}^{2}}=q-1 \geq 0$ and $p+q=n-2$, then $P_{k+1}^{n}\left(a_{2}, a_{3}, \ldots, a_{k}\right)$ is a double star and denoted by $D S_{n}(p, q)$ for short. And $P_{k+1}^{n}\left(a_{2}, a_{3}, \ldots, a_{k}\right)$ is a dumbbell and denoted by $D B_{n}\left(a_{2}, a_{k}\right)$ if $a_{2} \geq 0, a_{k} \geq 0$ and $a_{t}=0$ for $3 \leq t \leq k-1$. Moreover, $P_{k+1}^{n}\left(a_{2}, a_{3}, \ldots, a_{k}\right)$ is called a volcano tree and denoted by $V_{n}(n-k-1)$ if $a_{t}=n-k-1$ when $v_{t}$ is a central vertex of $P_{k+1}$ and $a_{j}=0$ for any $j \neq t$ for even $k$, and in the set $\mathcal{V}_{n}(n-k-1)$ if $a_{t}+a_{t+1}=n-k-1$ when $v_{t}, v_{t+1}$ are two central vertices of $P_{k+1}$ and $a_{j}=0$ for any $j \neq t, t+1$ for odd $k$. If $d=2$, $\mathcal{T}_{n}(d)$ contains a single tree $S_{n}$. The case is same when $n=3$. For $n=4$, there are exactly two trees $S_{n}$ and $P_{n}$ with $E_{1}\left(P_{n}\right)>\xi^{c}\left(P_{n}\right)$ and $E_{1}\left(S_{n}\right)>\xi^{c}\left(S_{n}\right)$. So in the following we always assume that $d \geq 3$ and $n \geq 5$. For convenience, here we set $\xi^{A}(G)=E_{1}(G)-\xi^{c}(G)$ for any connected graph $G$.

Lemma 2.1. Suppose that $T \in \mathcal{T}_{n}(d)$ with $n \geq 5$ and $3 \leq d<n-1$ minimizes the value of $\xi^{A}$. Then T must be a caterpillar.

Proof. We choose an arbitrary tree $T \in \mathcal{T}_{n}(d)$ with $\xi^{A}(T)$ as small as possible. If $T \cong P_{d+1}^{n}\left(a_{2}, a_{3}, \ldots, a_{d}\right)$, then our result holds immediately. Otherwise, we can assume that $P_{d+1}=$ $v_{1} v_{2} \cdots v_{d} v_{d+1}$ is a diametral path in $T$. Then $T$ can be viewed as a tree obtained by attaching a subtree $T_{i}$ to each of vertex $v_{i}$ with $i \in\{2,3, \ldots \ldots, d\}$ such that $\operatorname{diam}\left(T_{i}\right) \leq$ $d-\varepsilon_{T}\left(v_{i}\right)$. There must be a pendant vertex, say $v_{k}$, from $V\left(T_{m}\right)$ with $d_{T}\left(v_{k}, v_{m}\right) \geq 2$ where $m \in\{3,4, \ldots, d-1\}$ and $v_{k} v_{j} \in E(T) \quad(j \notin\{1,2, \ldots, d, d+1\})$. Without loss of generality, we assume that $\varepsilon_{T}\left(v_{m}\right)=d_{T}\left(v_{m}, v_{d+1}\right)=d+1-m$ and $\varepsilon_{T}\left(v_{k}\right)=t$ with $t>d+1-m+1$.

Now we construct a new tree $T^{\prime}$ obtained from $T$ by deleting the edge $v_{k} v_{j}$ and adding a new edge $v_{k} v_{m}$. Then $T^{\prime}$ still belongs to $\mathcal{T}_{n}(d)$ with $\varepsilon_{T^{\prime}}\left(v_{k}\right)=d+1-m+1$ with $\varepsilon_{T^{\prime}}\left(v_{m}\right)=d+1-m$ and $\varepsilon_{T^{\prime}}\left(v_{x}\right)=\varepsilon_{T}\left(v_{x}\right)$ where $x \neq k$. Note that $t>d+1-m+1$. Therefore, only considering the contribution of the vertices $v_{j}, v_{k}$ and $v_{m}$ to $\xi^{A}$, we have

$$
\begin{aligned}
\xi^{A}(T)-\xi^{A}\left(T^{\prime}\right)= & {\left[t-1-\operatorname{deg}_{T}\left(v_{j}\right)\right](t-1)+t(t-1) } \\
& +(d+1-m)\left[d+1-m-\operatorname{deg}_{T}\left(v_{m}\right)\right] \\
& -\left[t-1-\left(\operatorname{deg}_{T}\left(v_{j}\right)-1\right)\right](t-1) \\
& -(d+2-m-1)(d+2-m) \\
& -(d+1-m)\left[d+1-m-\left(\operatorname{deg}_{T}\left(v_{m}\right)+1\right)\right]
\end{aligned}
$$




$$
\begin{aligned}
& =-(t-1)+(d+1-m)+t(t-1) \\
& -(d+1-m)(d+2-m) \\
& =(t-1)^{2}-(d+1-m)^{2}>0,
\end{aligned}
$$

that is, $\xi^{A}\left(T^{\prime}\right)<\xi^{A}(T)$. If $T^{\prime}$ is a caterpillar, our result follows. If not, we can continue the above construction process until we obtain a caterpillar $P_{d+1}^{n}\left(a_{2}, a_{3}, \ldots, a_{d}\right) \in \mathcal{T}_{n}(d)$. Then our result holds from the fact that $\xi^{A}$ strictly decreases in the above construction process.

Lemma 2.2. Let $T \cong P_{d+1}^{n}\left(a_{2}, a_{3}, \ldots, a_{d}\right)$ be a non-volcano tree. Then there is another caterpillar $T^{\prime} \in \mathcal{T}_{n}(d)$ with $\xi^{A}\left(T^{\prime}\right)<\xi^{A}(T)$.

Proof. By assumption, there is a non-central vertex $v_{i}$ in the diametral path of $T \cong P_{d+1}^{n}\left(a_{2}, a_{3}, \ldots, a_{d}\right)$ such that $a_{i}>0$. First, let $d$ be even. When $d$ is odd, our proof can be similarly completed and so omitted here. Without loss of generality, assume that $i<\frac{d}{2}$. Now we construct a new tree $T^{\prime}$ from $T$ by deleting all the pendant edges incident with $v_{i}$ and joining them with the vertex $v_{i+1}$. Note that $\operatorname{deg}_{T}\left(v_{i}\right)=a_{i}+2$ and $T^{\prime}$ is still a caterpillar with diameter d. Moreover, we have

$$
\begin{aligned}
& \xi^{A}(T)-\xi^{A}\left(T^{\prime}\right) \\
& =a_{i}[(d-i+2)(d-i+2-1)-(d-i+1)(d-i+1-1)] \\
& \quad+(d-i+1)\left[\left(d-i+1-a_{i}-2\right)-(d-i+1-2)\right] \\
& \quad+(d-i)\left[\left(d-i-\operatorname{deg}_{T}\left(v_{i+1}\right)\right)-\left(d-i-\operatorname{deg}_{T}\left(v_{i+1}\right)-a_{i}\right)\right] \\
& =a_{i}\left[(d-i+2)^{2}-(d-i+1)^{2}-1\right]-a_{i}(d-i+1)+a_{i}(d-i) \\
& =a_{i}[2(d-i)+1] \\
& >0,
\end{aligned}
$$

which finishes the proof of this lemma.

Now we define the a function as follows:

$h(n, d)= \begin{cases}2 \sum_{i=\frac{d+1}{2}}^{\delta}\left(i^{2}-2 i\right)+\frac{(d+1)}{4}[(n-d-2) d+n]+\frac{7 d-1}{4} \text { if } d \text { is odd } ; \\ 2 \sum_{\substack{i=\frac{d}{2} \\ \delta}}\left(i^{2}-2 i\right)+\frac{d^{2}(n-d-2)}{4}+3 d & \text { if } d \text { is even. }\end{cases}$

By some calculations, we have $\xi^{A}\left(V_{n}(n-d-1)\right)=h(n, d)$ for even $d$ and $\xi^{A}(T)=h(n, d)$ for any $T \in \mathcal{V}_{n}(n-d-1)$ when $d$ is odd.

Theorem 2.3. Let $T$ be a tree of order $n \geq 4$. Then

$$
\xi^{A}(T) \geq n
$$

with equality holding if and only if $T \cong S_{n}$.

Proof. Note that the set of all trees of order $n$ can be partitioned into the union of the sets $\mathcal{T}_{n}(d)$ with $d \in\{2,3, \ldots, n-1\}$. Moreover, it can be easily checked that $h(n, d)>h(n, d-2)$ for any polarity of $d$.

Assume that $T$ is a tree of order $n \geq 3$ with $\xi^{A}$ as small as possible. By the above argument, we conclude that $T$ is a tree in $\mathcal{T}_{n}(2)$ or in $\mathcal{T}_{n}(3)$, that is, $T \cong S_{n}$ or $T \cong D S_{n}\left(n_{1}, n_{2}\right)$ with $n_{1} \geq 1, n_{2} \geq 1$ and $n_{1}+n_{2}=n-2$. By definition, we have $\xi^{A}\left(S_{n}\right)=n<4 n-4=\xi^{A}\left(D S_{n}\left(n_{1}, n_{2}\right)\right)$. Thus $T \cong S_{n}$ from the choice of $T$.

Conversely, if $T \cong S_{n}$, we have $\xi^{A}(T)=n$, finishing the proof of this theorem.

From Theorem 2.3, the following corollary can be easily obtained.

Corollary 2.4. Let $T$ be a tree of order $n \geq 3$. Then $E_{1}(T)>\xi^{c}(T)$.

From Corollary 2.4, any chemical tree $T$ fulfills the property that $E_{1}(T)>\xi^{c}(T)$, which is also recently proved by Das. ${ }^{[25]}$

Note that any vertex in the cycle $C_{n}$ has the same eccentricity $\left\lfloor\frac{n}{2}\right\rfloor$. Therefore we have $E_{1}\left(C_{n}\right)>\xi^{c}\left(C_{n}\right)$ for $n>4$. In the following theorem we prove the existence of chemical graphs $G$ with $E_{1}(G)>\xi^{c}(G)$.

Theorem 2.5. There are infinite number of chemical graphs $G$ such that $E_{1}(G)>\xi^{c}(G)$.

Proof. Now we consider the graph $G=C_{n} \square K_{2}$ with $n \geq 6$. Note that $G$ is a 3-regular graph of order $2 n$. Assume that $V(G)=\left\{v_{1}, v_{2}, \ldots, v_{n}, u_{1}, u_{2}, \ldots, u_{n}\right\} \quad$ where $v_{1} v_{2} \ldots v_{n}$ and $u_{1} u_{2} \ldots u_{n}$ with their natural adjacency relation form two induced cycle $C_{n}$ in $G$.

For any positive integer $k$ with $1 \leq k \leq n$, we set $|k|_{n}=\min \{k, n-k\}$. It can be verified that $\varepsilon_{G}\left(v_{i}\right)=\left\lfloor\frac{n}{2}\right\rfloor+1>3$ for any vertex $v_{i}$ with $u_{t}$ as its eccentric vertex in $G$ where $|t|_{n}=\left\lfloor\frac{n}{2}\right\rfloor$. By symmetry, we have $\varepsilon_{G}\left(u_{j}\right)=\left\lfloor\frac{n}{2}\right\rfloor+1$ for any vertex $u_{j}$. Thus $\xi^{A}(G)>0$, that is, $E_{1}(G)>\xi^{c}(G)$ as desired.

\section{THE GRAPHS WITH $E_{1}(G) \leq \xi^{c}(G)$}

In this section we prove several results on the graphs $G$ with $E_{1}(G) \leq \xi^{c}(G)$.

Recall that $E_{1}\left(K_{2}\right)=\xi^{c}\left(K_{2}\right)$. Also $E_{1}\left(C_{4}\right)=\xi^{c}\left(C_{4}\right)$ with $C_{4}=K_{2}^{(2)}$. Note that, for $k \geq 2, K_{2}^{(k)}$ is just the $k$-cube which is a $k$-regular graph with each vertex with eccentricity k. Now we give a more general result.

Example 3.1. $E_{1}\left(K_{2}^{(k)}\right)=\xi^{c}\left(K_{2}^{(k)}\right)$ for any $k \geq 1$.

Next we turn to the results for the graphs $G$ with $E_{1}(G)<\xi^{c}(G)$. Although in Section 2 we prove that $E_{1}(T)>\xi^{c}(T)$ for any tree of order $n>2$, we have the opposite result for the complements of all trees of order $n>2$. Below we first list an essential lemma for the complement of a graph. 
Lemma 3.2. ${ }^{[27]}$ Let $G$ be a connected graph with the connected complement.

(i) If $d>3$, then $\bar{G}$ has diameter $\bar{d}=2$.

(ii) If $d=3$, then $\bar{G}$ has a spanning subgraph which is a double star.

Lemma 3.3. Let $G$ be a self-centered graph of order $n>2$ with $v_{i} \in V(G)$. Then $\operatorname{deg}_{G}\left(v_{i}\right) \geq 2$.

Proof. To the contrary, we assume that $\operatorname{deg}_{G}\left(v_{i}\right)=1$ with $v_{i} v_{j} \in E(G)$. Note that $n>2$. Then $\varepsilon_{G}\left(v_{i}\right)=\varepsilon_{G}\left(v_{j}\right)+1$. This is a contradiction since $G$ is self-centered.

Note that $\overline{P_{3}}$ is disconnected and $\overline{P_{4}}=P_{4}$. Moreover, it can be verified that $E_{1}(\bar{T})<\xi^{c}(\bar{T})$ for the trees of order 5 except $D S_{5}(1,2)$ with $E_{1}\left(\overline{D S_{5}(1,2)}\right)>\xi^{c}\left(\overline{\left.D S_{5}(1,2)\right)}\right.$. So we assume that $n>5$ in the following theorem.

Theorem 3.4. Let $T$ be a tree of order $n>5$ with diameter $d>2$. Then $E_{1}(\bar{T})<\xi^{c}(\bar{T})$.

Proof: If $d=3$, then $T \cong D S_{n}\left(n_{1}, n_{2}\right)$ with $n_{1}+n_{2}=n-2>2$, $n_{1} \geq 1$ and $n_{2} \geq 1$. Assume that the only two vertices with eccentricity 2 in $T$ are $v_{1}$ and $v_{2}$ with $\operatorname{deg}_{T}\left(v_{1}\right)=$ $n_{1}+1 \leq n_{2}+1=\operatorname{deg}_{T}\left(v_{2}\right)$. Then $\varepsilon_{\bar{T}}\left(v_{1}\right)=\varepsilon_{\bar{T}}\left(v_{2}\right)=3$, and all other vertices have the same eccentricity 2 in $\bar{T}$. Moreover, all vertices other than $v_{1}, v_{2}$ in $\bar{T}$ have degrees $n-2$. If $n_{1}=1$, then $n_{2}=n-3$ and $\operatorname{deg}_{\bar{T}}\left(v_{1}\right)=n-3$, $\operatorname{deg}_{\bar{T}}\left(v_{2}\right)=1$. Note that $n>5$. Then we have

$$
\begin{aligned}
\xi^{c}(\bar{T})-E_{1}(\bar{T}) & =3(n-3-3)+3(1-3)+2(n-2)(n-2-2) \\
& =2 n^{2}-9 n-8>0 .
\end{aligned}
$$

For $n_{1} \geq 2$, similarly as above, we get

$$
\xi^{c}(\bar{T})-E_{1}(\bar{T})=2 n^{2}-9 n-8>0 .
$$

If $d>3$, then, by Lemma $3.2(i), \bar{T}$ has diameter $\bar{d}=2$. Now we claim that $\bar{T}$ is a 2 -self-centered graph. If not, $\bar{T}$ has a vertex $v_{i}$ with $\varepsilon_{\bar{T}}\left(v_{i}\right)=1$, i.e., $\operatorname{deg}_{\bar{T}}\left(v_{i}\right)=n-1$. Thus $v_{i}$ is an isolated vertex in T, which is a contradiction since $T$ is a tree. Observe that a connected 2-regular graph is just a cycle $C_{n}$ whose complement is not a tree for $n>5$, in view of Lemma 3.3, we find that $\bar{T}$ is a 2-self-centered graph with $\operatorname{deg}_{\bar{T}}\left(v_{i}\right) \geq 2$ for any vertex $v_{i}$ and there is at least one vertex $v_{j}$ with $\operatorname{deg}_{\bar{T}}\left(v_{j}\right)>2$. Thus $\xi^{c}(\bar{T})-E_{1}(\bar{T})>0$, finishing the proof of the theorem.

In the following theorem we give a sufficient condition for the graphs $G$ of order $n$ and with $E_{1}(G)<\xi^{c}(G)$.

Theorem 3.5. Let $G$ be a connected graph of order $n \geq 5$ with $K_{2, n-2}$ as its subgraph. Then $E_{1}(G)<\xi^{c}(G)$.

Proof. If $G \cong K_{2, n-2}$, then we have $\xi^{c}(G)=8(n-2)$ and $E_{1}(G)=4 n$. It follows that $\xi^{c}(G)>E_{1}(G)$ from $n \geq 5$. Therefore in the following we assume that $G$ contains $K_{2, n-2}$ as a proper spanning subgraph.
Since $G$ contains $K_{2, n-2}$ as a proper spanning subgraph, the eccentricity of any vertex in $G$ is 1 or 2. Moreover, $\operatorname{deg}_{G}\left(v_{k}\right) \geq 2$ for any vertex $v_{k} \in V(G)$ with $\varepsilon_{G}\left(v_{k}\right)=2$, and $d_{G}\left(v_{i}\right)=n-1$ for any vertex $v_{i} \in V(G)$ with $\varepsilon_{G}\left(v_{i}\right)=1$. If there is a vertex $v_{k} \in V(G)$ with degree $n-1$, then $\xi^{c}(G)>E_{1}(G)$ immediately. Otherwise, $G$ is 2-self-centered graph. Considering that $K_{2, n-2}$ is a proper spanning subgraph of $G$, there are at least two vertices $v_{i}, v_{j}$ with $\operatorname{deg}_{G}\left(v_{i}\right)>\varepsilon_{G}\left(v_{i}\right)$ and $\operatorname{deg}_{G}\left(v_{j}\right)>\varepsilon_{G}\left(v_{j}\right)$. So $\xi^{c}(G)>E_{1}(G)$, finishing the proof of the theorem.

We can easily observe that the graph $G$ in Theorem 3.5 has minimum degree at least 2 and $\operatorname{deg}_{G}\left(v_{i}\right) \geq \varepsilon_{G}\left(v_{i}\right)$ for any vertex $v_{i} \in V(G)$ with at least one strict inequality. But these conditions are not necessary for $\xi^{c}(G)>E_{1}(G)$. Next we will give an example with $\xi^{c}(G)>E_{1}(G)$ but not satisfying these above conditions. Denote by $G^{*}$ the graph obtained by attaching a pendant vertex to each of the vertices in a connected graph $G$. Clearly, for $n \geq 3$, any pendant vertex in $K_{n}^{*}$ has eccentricity 3 , and any other vertex has a same eccentricity 2 in it. Thus $\xi^{c}\left(K_{n}^{*}\right)-E_{1}\left(K_{n}^{*}\right)=$ $2 n(n-2)+3 n(1-3)=2 n(n-5)>0$ if $n>5$.

Example 3.6. $E_{1}\left(K_{n}^{*}\right)<\xi^{c}\left(K_{n}^{*}\right)$ for $n>5$.

Note that all the graphs described in Theorems 3.4 and 3.5 have diameter at most 3. In Ref. [28], a class of graphs are constructed with exactly two distinct eccentricities (see Figure 1). Here $G_{0}$ is an arbitrary graph, each of whose vertices is adjacent to any vertex from $\left\{x_{1}, y_{1}, z_{1}, w_{1}\right\}$. The black vertices have eccentricity $r-1$ and the white vertices have eccentricity $r$ in $\mathrm{G}$. The graph schematically shown in Figure 1 is denoted by $G=S\left(G_{0} ; r-1, r\right)$ with diameter $r$. In the following theorem we prove the existence of graphs $G$ with diameter more than 2 fulfilling $E_{1}(G)<\xi^{c}(G)$.

Theorem 3.7. For any integer $r \geq 2$, there is a graph $G$ with diameter $r$ and fulfilling $E_{1}(G)<\xi^{c}(G)$.

Proof. For any integer $r \geq 2$, we choose a graph $G=$ $S\left(K_{n} ; r-1, r\right)$ with $n \geq 3 r$. From the structure of $S\left(K_{n} ; r-1, r\right)$, we find that there are $n$ vertices in $K_{n}$ of $S\left(K_{n} ; r-1, r\right)$ with eccentricity $r-1$ and degree $n+3$, other vertices have a

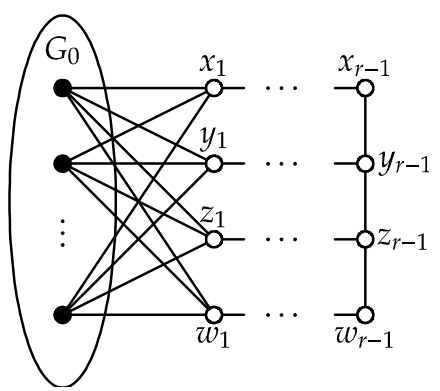

Figure 1. Graph $G$ with only two eccentricities $r-1$ and $r$. 
same eccentricity $r$ in $S\left(K_{n} ; r-1, r\right)$. Note that $n \geq 3 r$ and $r \geq 2$. Then

$$
\begin{aligned}
\xi^{c}(G)-E_{1}(G)= & {[n+3-(r-1)] n(r-1)+(3-r) 2 r } \\
& +(2-r)[4(r-3)+2] r+(n+1-r) 4 r \\
= & (n+4-r) n(r-1)+(n+1-r) 4 r \\
& -2 r\left(2 r^{2}-8 r+7\right) \\
\geq & 3 r(2 r+4)(r-1)+(2 r+1) 4 r \\
& -2 r\left(2 r^{2}-8 r+7\right) \\
= & 2 r\left(r^{2}+15 r-11\right)>0 .
\end{aligned}
$$

This completes the proof of the theorem.

\section{AN EFFECTIVE CONSTRUCTION}

From Example 3.1, we would like to give a more general result for generating infinite graphs with different comparison relations. To do it, we first prove a useful lemma as follows.

Lemma 4.1. Let $G$ be a connected graph of order $n>2$ and $H=G \square K_{2}$. Then $\varepsilon_{H}(w)=\varepsilon_{G}(w)+1$ for any vertex $w \in V(H)$.

Proof. Assume that $V(G)=\left\{v_{1}, v_{2}, \ldots, v_{n}\right\}$ and $V(H)=\left\{v_{1}, v_{2}\right.$, $\left.\ldots, v_{n}, v_{1}^{\prime}, v_{2}^{\prime}, \ldots, v_{n}^{\prime}\right\}$ with $H\left[\left\{v_{1}, v_{2}, \ldots, v_{n}\right\}\right]=H\left[\left\{v_{1}^{\prime}, v_{2}^{\prime}, \ldots, v_{n}^{\prime}\right\}\right] \cong$ $G$ where $v_{i}^{\prime}$ is the copy of vertex $v_{i}$ in $H$ for $i=1,2, \ldots, n$. By symmetry, it suffices to prove that $\varepsilon_{H}\left(v_{i}\right)=\varepsilon_{G}\left(v_{i}\right)+1$ for $i=1,2, \ldots, n$.

Assume that $\varepsilon_{G}\left(v_{i}\right)=k$ for an arbitrary vertex $v_{i} \in V(G) \subseteq V(H)$. Then there exists a vertex $v_{j}$ as an eccentric vertex of $v_{i}$ in $G$. Therefore $\varepsilon_{H}\left(v_{i}\right) \geq d_{H}\left(v_{i}, v_{j}^{\prime}\right)=$ $k+1$ from the structure of $H$. Next we prove that $\varepsilon_{H}\left(v_{i}\right) \leq k+1$. Otherwise, we have $\varepsilon_{H}\left(v_{i}\right) \geq k+2$. Then there is a vertex $v_{m}^{\prime} \in V(H) \backslash V(G)$ with $d_{H}\left(v_{i}, v_{m}^{\prime}\right) \geq k+2$. By the structure of $H$, again, we have $d_{G}\left(v_{i}, v_{m}\right) \geq k+1$, contradicting the fact that $\varepsilon_{G}\left(v_{i}\right)=k$. So $\varepsilon_{H}\left(v_{i}\right)=k+1=$ $\varepsilon_{G}\left(v_{i}\right)+1$, finishing the proof of the lemma.

Theorem 4.2. Assume that $G$ is a connected graph of order $n \geq 2$ with $\varepsilon_{G}\left(v_{i}\right) \geq \operatorname{deg}_{G}\left(v_{i}\right)$ for any vertex $v_{i} \in V(G)$. Then $E_{1}\left(G \square K_{2}\right) \geq \xi^{c}\left(G \square K_{2}\right)$.

Proof. Let $H=G \square K_{2}$. From the structure of $H$, we have $\operatorname{deg}_{H}(w)=\operatorname{deg}_{G}(w)+1$ for any vertex $w \in V(G)$. Thus $E_{1}(G) \geq \xi^{c}(G)$. By Lemma 4.1, we have

$$
\begin{aligned}
& E_{1}\left(G \square K_{2}\right)-\xi^{c}\left(G \square K_{2}\right) \\
& =2 \sum_{v_{i} \in V(G)}\left(\varepsilon_{G}\left(v_{i}\right)+1\right)\left[\left(\varepsilon_{G}\left(v_{i}\right)+1\right)-\left(\operatorname{deg}_{G}\left(v_{i}\right)+1\right)\right] \\
& \geq 4\left(E_{1}(G)-\xi^{c}(G)\right) \geq 0 .
\end{aligned}
$$

Therefore, our result holds immediately.
Similarly as above, we can easily obtain the corollary below.

Corollary 4.3. Assume that $G$ is a connected graph of order $n>2$ with $\varepsilon_{G}\left(v_{i}\right)<\operatorname{deg}_{G}\left(v_{i}\right)$ for any vertex $v_{i} \in V(G)$. Then $E_{1}\left(G \square K_{2}\right)<\xi^{c}\left(G \square K_{2}\right)$.

By Theorem 4.2 and Corollary 4.3, we can get infinite graphs with each possibility for comparison between the first Zagreb eccentricity index and eccentric connectivity index.

Recall that the graph $G^{*}$ is defined in Section 3. In addition to Example 3.1 for $E_{1}=\xi^{c}$, by Theorem 4.2 and Corollary 4.3, we can give the following examples for other comparative inequalities between $E_{1}$ and $\xi^{c}$.

Example 4.4. $E_{1}\left(C_{n}^{*} \square K_{2}\right)>\xi^{c}\left(C_{n}^{*} \square K_{2}\right)$ for any $n \geq 4$, $E_{1}\left(K_{n_{1}, n_{2}} \square K_{2}\right)<\xi^{c}\left(K_{n_{1}, n_{2}} \square K_{2}\right)$ for $n_{2} \geq n_{1}>2$.

\section{CONCLUSION}

In this paper we present some results on the comparison between $E_{1}(G)$ and $\xi^{c}(G)$ on the graphs $G$ including some chemical graphs. In particular, we show that $E_{1}(T)>\xi^{c}(T)$ for any trees $T$ including chemical trees. Some sufficient conditions are obtained on the graphs $G$ with $E_{1}(G) \leq \xi^{c}(G)$. Moreover, we also give a construction method for generating infinite graphs $G$ for each comparative inequality between $E_{1}(G)$ and $\xi^{c}(G)$. Now it seems to be an open and attractive problem to characterize completely chemical graphs with some comparative inequality between $E_{1}$ and $\xi^{c}$.

Acknowledgment. The authors thank three anonymous referees for their careful reading and helpful comments on our paper. The authors are supported by NNSF of China (No. 11671202) and Chinese Excellent Overseas Researcher Funding in 2016.

\section{REFERENCES}

[1] J. A. Bondy and U. S. R. Murty, Graph Theory with Applications, Macmillan Press, New York, 1976.

[2] I. Gutman, N. Trinajstić, Chem. Phys. Lett. 1972, 17, 535.

[3] I. Gutman, B. Ruščić, N. Trinajstić, C. F.Wilcox, J. Chem. Phys. 1975, 62, 3399.

[4] A. T. Balaban, I. Motoc, D. Bonchev, O. Mekenyan, Topics Curr. Chem. 1983, 114, 21.

[5] S. Nikolić, G. Kovačević, A. Miličević, N. Trinajstić, Croat. Chem. Acta 2003, 76, 113.

[6] K. Xu, Appl. Math. Lett. 2011, 24, 1026.

[7] K. Xu, K. C. Das, S. Balachandran, MATCH Commun. Math. Comput. Chem. 2014, 72, 641.

[8] I. Gutman, Bulletin of Society of Mathematicians Banja Luka 2011, 18, 17. 
[9] K. Xu, H. Hua, MATCH Commun. Math. Comput. Chem. 2012, 68, 241.

[10] M. Eliasi, A. Iranmanesh, I. Gutman, MATCH Commun. Math. Comput. Chem. 2012, 68, 217.

[11] K. Xu, K. C. Das, MATCH Commun. Math. Comput. Chem. 2012, 68, 257.

[12] A. R. Ashrafi, T. Došlić, A. Hamzeh, Disc. Appl. Math. 2010, 158, 1571.

[13] A. R. Ashrafi, T. Došlić, A. Hamzeh, MATCH Commun. Math. Comput. Chem. 2011, 65, 85.

[14] D. Vukičević and A. Graovac, Acta Chim. Slov. 2010, 57, 524.

[15] K. C. Das, D.W. Lee, A. Graovac, Ars Mathematica Contemporanea 2013, 6, 117.

[16] Z. Du, B. Zhou, N. Trinajstić, Croat. Chem. Acta 2012, 85, 359.

[17] V. Sharma, R. Goswami, A. K. Madan, J. Chem. Inf. Comput. Sci. 1997, 37, 273.

[18] H. Dureja, S. Gupta, A. K. Madan, J. Mol. Graph. Model. 2008, 26, 1020.
[19] S. Sardana, A. K. Madan, MATCH Commun. Math. Comput. Chem. 2001, 43, 85.

[20] S. Sardana, A. K. Madan, MATCH Commun. Math. Comput. Chem. 2002, 45, 35.

[21] A. Ilić, I. Gutman, MATCH Commun. Math. Comput. Chem. 2011, 65, 731.

[22] M. J. Morgan, S. Mukwembi, H. C. Swart, Discrete Math. 2011, 311, 1229.

[23] K. Xu, K. C. Das, H. Liu, J. Math. Anal. Appl. 2016, 433, 803.

[24] I. Gutman, O. E. Polansky, Mathematical Concepts in Organic Chemistry, Springer, Berlin, 1986.

[25] K. C. Das, Croat. Chem. Acta 2016, 89, in press.

[26] F. Harary, A. J. Schwenk, Discrete Math. 1973, 6, 359.

[27] L. Zhang, B. Wu, MATCH Commun. Math. Comput. Chem. 2005, 54, 189.

[28] S. Klavžar, H. Liu, P. Singh, K. Xu, Constructing almost peripheral and almost self-centered graphs revisited, Taiwanese J. Math., in press. 